\title{
Possible Role of BCG-Induced Trained Immunity to Reduce the Prevalence and Severity of COVID-19 in High-Risk Population
}

\section{Gupta $\mathrm{G}^{1}$, Chatterji $\mathrm{S}^{1}$, Thukral $\mathrm{H}^{1}$, Arumugaswami $\mathrm{V}^{2}$, Guha $\mathrm{SK}^{3}$, Basu $\mathbf{A}^{3}$, Gupta $S^{4}$ and Sinha $\mathbf{S}^{1 *}$}

${ }^{1}$ Department of Medicine, All India Institute of Medical Sciences, New Delhi, India

${ }^{2}$ University of California, Los Angeles, USA

${ }^{3}$ School of Tropical Medicine, Kolkata, India

${ }^{4}$ Sanjay Gandhi Postgraduate Institute of Medical Sciences, Lucknow, India

\section{Review Article}

Volume 4 Special Issue 1

Received Date: July 06, 2020

Published Date: July 29, 2020

DOI: $10.23880 /$ jidtm-16000S1-005

*Corresponding author: Sanjeev Sinha, Professor of Medicine, Department of Medicine, All India Institute of Medical Sciences, New Delhi, India, Tel: +91-9810164416, Fax: +91-11-26588866; Email: drsanjeevsinha@gmail.com

\section{Abstract}

In the last few months COVID-19 has been spreading unchecked to most of the countries of the world. While the impact has varied from one nation to the other and the differences attributed to various factors, universal BCG vaccination is thought to be protective by some. BCG is thought to protect by the new concept of trained immunity over and above the enhanced innate and adaptive immune response. In the current scenario, the most effective way to curb the spread of the virus is a vaccine and it may take at least 12-18 months to develop. In the meantime, other measures like hand hygiene, mask use, social distancing, and lockdown are being implemented to prevent the spread of the virus. We present here a short review on the protective role of BCG in COVID-19.

Keywords: BCG vaccination; Heterologous; Droplet infection; Cytokines and chemokines; Immunity

Abbreviations: BCG: Bacille Calmette-Guerin; COVID-19: Coronavirus Disease; HCW: Health Care Workers; ACE2: Angiotensin-Converting Enzyme 2; DCs: Dendritic Cells; TLRs: Toll-Like Receptors; APC: Antigen-Presenting Cell; IL: Interleukin; CRS: Cytokine Release Syndrome; HLH: Hemophagocytic Lymphohistiocytosis; TNF- $\alpha$ : Tumor Necrosis Factor-alpha; PRRs: Pattern Recognition Receptors.

\section{Introduction}

In the last few months COVID-19 has been spreading unchecked to most of the countries of the world. The impact has varied from one nation to the other. These differences may be attributed to differences in culture, mitigation efforts, and healthcare infrastructure. Studies suggest high-risk population which includes older adults (more than 60 years of age), and adults with comorbidities (like cardiovascular diseases, cerebrovascular diseases, diabetes, chronic lung disease, chronic kidney diseases, chronic liver diseases, and malignancy) are at higher risk of SARS-CoV-2 infectionrelated morbidity and mortality [1].

An epidemiological study done by Miller A, et al. [2] concluded that the countries without universal policies of BCG vaccination like Italy, Netherlands, and United states of America had grave outcomes compared to countries with universal and long-standing BCG policies. This study also concluded that the countries which had a late start of universal BCG policy like Iran had higher mortality in the elderly population [2]. Studies have shown that BCG vaccination produces non-specific protection against viral infections and sepsis, leading to the hypothesis of the 
protective effect of BCG against COVID-19 [3]. The vaccine has shown heterologous protective effects and decreased overall childhood mortality. Some epidemiological studies propose that differences in COVID-19 impact can be partially explained by the differences in national policies concerning for BCG vaccination. The unintended health benefits that have been observed are risk reductions for acute respiratory infections, cancer, asthma, and overall childhood mortality [4-7].

The association between the universal BCG vaccination and the protection against COVID-19 suggests that BCG may confer long-lasting protection against the current strain of SARS-CoV-2. Currently three clinical trials are ongoing to evaluate the role of BCG vaccine in protecting health care workers (HCW) against COVID-19. All the clinical trials namely, BRACE (NCT04327206), BADAS (NCT04348370), and BCG-CORONA (NCT04328441) are assessing the ability of BCG vaccine to mitigate the incidence and severity of COVID-19 [8-10].

In the current scenario, the most effective way to curb the spread of the virus is a vaccine and it may take at least 12-18 months to develop [11]. In the meantime, other measures like hand hygiene, mask use, social distancing, and lockdown are being implemented for preventing the spread of the virus.

\section{Immunopathogesis of SARS-CoV-2}

\section{Mechanism of Infection and Immune Invasion}

SARS-CoV-2 is the seventh known coronavirus in humans and belongs to the same phylogenetic family as the 2002 SARS and the 2012 MERS-CoV-2 [12]. The major route of transmission of the virus is through droplet infection and close person-to-person contact. However, some studies have reported transmission through sweat, stool, and urine [13]. The cycle of infection, replication, and transmission requires a susceptible host and adequate environment. The primary target cells of the virus in the body are enterocytes and pneumocytes. There are five main steps in the life cycle of the virus: attachment, penetration, biosynthesis, maturation and release. The invasion starts with attachment of the viral structural spike (S) protein to the angiotensin-converting enzyme 2 (ACE2) receptor on the target cells [14]. The entry of the virus is either via clathrin-dependent or independent endocytosis. The fusion of viral and host cell membranes occurs via host type 2 transmembrane serine protease (TMPRSS2), this facilitates the release of viral nucleoprotein into the host cell. After the entry, the virus then orchestrates the synthesis of viral protein and genome polyproteins, subsequently leading to viral replication and final release of virus from the cell [12]. ACE2 receptors are widely expressed in lung (most prominent), heart, ileum, kidney, and bladder [15].

\section{Host Response to SARS-CoV-2}

In any infection, an adequate host response with the help of cytokines and chemokines usually negates any infection. Since ACE2 receptors are predominantly expressed on the apical side of lung epithelial cells in the alveolar space, thus viral-induced lung injury is predominantly in the lower respiratory tract. In airways, there are mainly three components of innate immunity, namely epithelial cells, alveolar macrophages and dendritic cells (DCs). SARSCoV-2 infection in the airways trigger a local anti-viral immune response, which recruits the macrophages and DCs. Ideally, SARS-CoV-2 RNA genome should trigger an antiviral response through the activation of pattern recognition receptors such as toll-like receptors (TLRs) which includes the release of pro-inflammatory cytokines and type 1 interferon [16].

The infected epithelial cells undergo apoptosis and are engulfed by macrophages and DCs which act as antigenpresenting cell (APC) thus activating cellular and humoral immunity to form virus-specific $\mathrm{T}$ cells and virus-specific antibody. As more and more pieces of evidence are generated, it appears that the asymptomatic, mild and non-severe cases of COVID-19 can generate an adequate anti-viral response as explained above. But in some cases, due to some risk factors still under research, there appears to be an immune dysregulation or exaggerated host response phase. This leads to an unchecked release of inflammatory cytokines such as interleukin (IL)-1ß, IL-6, monocyte chemoattractant protein 1 and may result in cytokine release syndrome (CRS) [17]. In severe COVID-19, patients have a characteristic finding of macrophage activation syndrome and immune dysregulation which is associated with sustained and hyperproduction of pro-inflammatory cytokines by monocytes, IL-6-mediated low HLA-DR expression, and lymphopenia [16,18,19]. All these lead to hyperactivation of the immune system which causes damage to the host and manifests as acute severe pneumonia, myocardial dysfunction, and secondary hemophagocytic lymphohistiocytosis (HLH), and eventually ends into multi-organ failure $[17,20,21]$. Till date, the exact mechanism of how the organism disrupts the host immune homeostasis and activates the hyperactive proinflammatory response is elusive [22]. A few hypotheses suggest an 'immune escape' mechanism adopted by SARS-Cov-2 which blinds the immune system causing an inadequate or delayed innate response [23]. BCG-induced trained immunity may enhance the innate immune response and this may be the possible mechanism to negate the immune escape of SARSCoV-2 [24]. 


\section{BCG-Induced Induced Immunity}

\section{Immune Response Induced by BCG Vaccination}

BCG antigens act as a benevolent vector due to proven safety in vaccinated neonates, children, and adults [25]. These antigens may act as adjuvants inducing innate and adaptive immunity. After the vaccination, at the site of inoculation, BCG antigens are recognized by the neutrophils, macrophages, and DCs. This activates DCs and migrates to lymph node secrete tumor necrosis factor-alpha (TNF- $\alpha$ ), interleukin 1-beta (IL-1 $\beta$ ), IL-6, and IL-12, further promotes the activation of adaptive immunity in form of CD4+(T helper cells) and CD8+ $\mathrm{T}$ cells(cytotoxic $\mathrm{T}$ cells). The Th1 subclass of CD4+ secrete IFN- $\gamma$, TNF- $\alpha$, and IL-2 thus contributes to the necessary activation of macrophage to kill microbes in the vicinity of macrophage $[24,26]$. CD8+ T cells secrete cytotoxins perforins, granzymes, and granulysin. After 10 weeks these $\mathrm{T}$ cells switch to a memory phenotype with a functional nature of secreting IFN- $\gamma$. These cells would generate a strong lymphoproliferative response to $\mathrm{TB}$ antigens or any new antigen in the future. Th2 subclass stimulates the B-cells into proliferation and induces a B-cell antibody class switching thus increase in production of IgG and memory B cells [27]. BCG provides non-specific protection against respiratory infections due to memory $\mathrm{B}$ and $\mathrm{T}$ cells, and long-term activation and reprogramming of innate immune cells thus, protecting against a wide range of organisms, including viruses such as influenza and yellow fever, bacteria such as Staphylococcus aureus, and fungi such as Candida albicans $[5,25,28]$.

\section{Trained Immunity as a Consequence of BCG}

Recently described is a memory phenotype in innate immune cells, known as "trained immunity", which is defined as an upsurge in non-specific response, mediated by innate immunity for an unrecognised or recognized infection [29]. This type of immunity is mediated primarily by monocytes/ macrophages and NK cells and independent of $\mathrm{T}$ and $\mathrm{B}$ cells responses (Figure 1). Post BCG vaccination, monocytes undergo epigenetic reprogramming, like methylation and acetylation of histone which enhances the chromatin accessibility and easier transcription of genes related to antimicrobial action. This epigenetic modification up regulates the expression of pattern recognition receptors (PRRs) that recognize specifically recognize pathogenassociated molecular patterns thus modulating the accessibility of transcription genes of proinflammatory cytokine genes. Thus, BCG vaccination of adults induces trained immunity in circulating monocytes/macrophages and increasing their capacity to produce pro-inflammatory cytokines such as IL-1 $\beta$, TNF- $\alpha$, and IL- 6 when exposed to a pathogen. Thus, the BCG vaccine may provide non-specific protection against respiratory infections due to memory $\mathrm{B}$ and $\mathrm{T}$ cells, and long-term activation and reprogramming of innate immune cells.

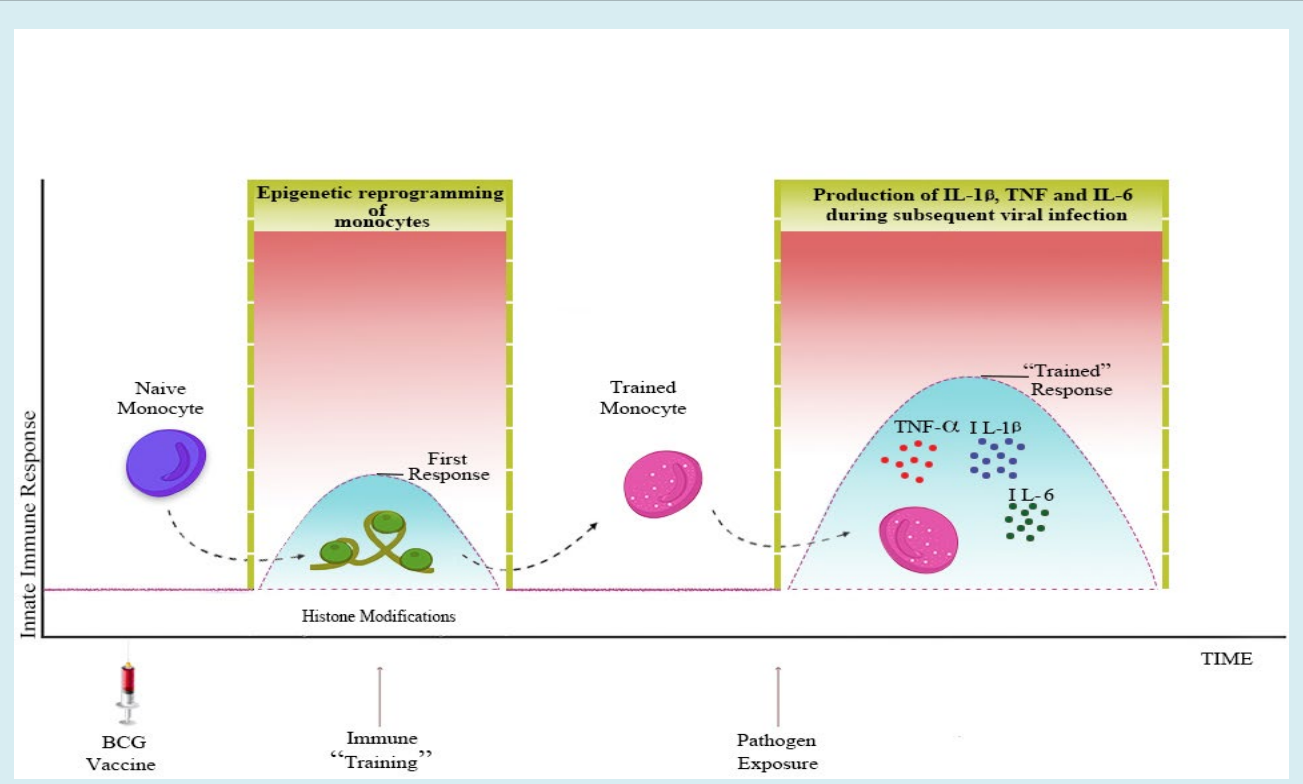

Figure 1: BCG vaccination induces trained immunity leading to enhanced innate immune response to pathogen. Thus, monocytes undergo epigenetic reprogramming, like methylation and acetylation of histone which enhances the chromatin accessibility and easier transcription of genes related to antimicrobial action. This is called "trained immunity" which enhances the effectiveness of the innate immune response when exposed to a non-specific pathogen, inducing the secretion of proinflammatory cytokines, such as TNF- $\alpha$, IL-1 $\beta$, and IL-6. The pink line represents a trained immune response [30]. 


\section{Possible Role of BCG to Induce 'Trained Immunity' in High Risk Groups}

Previous studies suggest adults with comorbidities are at higher risk of SARS-CoV-2 infection-related complications. These comorbidities are associated with alterations in the proliferation of $\mathrm{T}$ cells and macrophages, and impairment in the function of NK cells and B cells, thus these lead to an abnormal innate and adaptive immunity. This could be a possible reason for higher mortality in individuals with comorbidities [31]. Ecological studies have suggested that countries with universal BCG vaccination policy for the population have a reduced number of cases and mortality $[2,3]$. In comparison to the elderly population which fails to maintain a pool of trained monocyte, children who have been BCG vaccinated are less susceptible to SARS-CoV-2 infection. BCG vaccination in the high-risk populations may offer protection against SARS-CoV-2 by boosting trained immunity, leading to inhibition of viral replication and reduction in viral load and thus inflammation and immune dysregulation $[24,30,32]$.

\section{Conclusion}

In the current COVID-19 pandemic, the BCG vaccine could be a worthy option to enhance nonspecific immune response that may protect against SARS-CoV-2 infection. Thus, a hypothesis that BCG vaccination might have a role in protecting the high-risk populations against SARS-CoV-2 infection. But well designed, large randomized controlled trials are needed to provide the answer to the above hypothesis.

Acknowledgement: I thank Atul Kumar, Animator, Neurosurgery Education and Training School, Department of Neurosurgery, All India Institute of Medical Sciences, New Delhi, who helped in developing of the figure in the article.

\section{References}

1. Shereen MA, Khan S, Kazmi A, Bashir N, Siddique R (2020) COVID-19 infection: Origin, transmission, and characteristics of human coronaviruses. J Adv Res 24: 91-98.

2. Miller A, Reandelar MJ, Fasciglione $K$, Roumenova V, Li Y, et al. (2020) Correlation between universal BCG vaccination policy and reduced morbidity and mortality for COVID-19: an epidemiological study. medRxiv.

3. Shet A, Ray D, Malavige N, Santosham M, Bar-Zeev N (2020) Differential COVID-19-attributable mortality and BCG vaccine use in countries. Infectious Diseases (except HIV/AIDS).
4. Hollm-Delgado MG, Stuart EA, Black RE (2014) Acute Lower Respiratory Infection among Bacille CalmetteGuérin (BCG)-Vaccinated Children. Pediatrics 133(1): 73-81.

5. Fine PEM (1995) Variation in protection by BCG: implications of and for heterologous immunity. The Lancet 346(8986): 1339-1345.

6. Wardhana, Datau EA, Sultana A, Mandang VVV, Jim E (2011) The efficacy of Bacillus Calmette-Guerin vaccinations for the prevention of acute upper respiratory tract infection in the elderly. Acta Medica Indones 43(3): 185-190.

7. Lalor $\mathrm{MK}$, Ben-Smith A, Gorak-Stolinska P, Weir RE, Floyd S, et al. (2009) Population differences in immune responses to Bacille Calmette-Guérin vaccination in infancy. J Infect Dis 199(6): 795-800.

8. BCG Vaccination to Protect Healthcare Workers against COVID-19 (BRACE). ClinicalTrails.gov.

9. BCG Vaccine for Health Care Workers as Defense against COVID 19 (BADAS). ClinicalTrails.gov.

10. Reducing Health Care Workers Absenteeism in Covid-19 Pandemic through BCG Vaccine (BCG-CORONA). ClinivalTrails.gov.

11. Mullard A (2020) COVID-19 vaccine development pipeline gears up. Lancet 395(10239): 1751-1752.

12. Li G, Fan Y, Lai Y, Han T, Li Z, et al. (2020) Coronavirus infections and immune responses. J Med Virol 92(4): 424-432.

13. Parasa S, Desai M, Chandrasekar VT, Patel HK, Kennedy KF, et al. (2020) Prevalence of Gastrointestinal Symptoms and Fecal Viral Shedding in Patients With Coronavirus Disease 2019: A Systematic Review and Meta-analysis. JAMA Netw Open 3(6): 2011335.

14. Ciaglia E, Vecchione C, Puca AA (2020) COVID-19 Infection and Circulating ACE2 Levels: Protective Role in Women and Children. Front Pediatr.

15. Zou X, Chen K, Zou J, Han P, Hao J, et al. (2020) Single-cell RNA-seq data analysis on the receptor ACE2 expression reveals the potential risk of different human organs vulnerable to 2019-nCoV infection. Front Med 14: 185192.

16. Lingeswaran M, Goyal T, Ghosh R, Suri S, Mitra P, et al. (2020) Inflammation, Immunity and Immunogenetics in COVID-19: A Narrative Review. Indian J Clin Biochem 35(3): 260-273. 


\section{Journal of Infectious Diseases \& Travel Medicine}

17. Shetty R, Ghosh A, Honavar SG, Khamar P, Sethu S (2020) Therapeutic opportunities to manage COVID-19/SARSCoV-2 infection: Present and future. Indian J Ophthalmol 68(5): 693-702.

18. Giamarellos-Bourboulis EJ, Netea MG, Rovina N, Akinosoglou K, Antoniadou A, et al. (2020) Complex Immune Dysregulation in COVID-19 Patients with Severe Respiratory Failure. Cell Host Microbe 27(6): 992-1000.

19. Ni L, Ye F, Cheng ML, Feng Y, Deng YQ, et al. (2020) Detection of SARS-CoV-2-Specific Humoral and Cellular Immunity in COVID-19 Convalescent Individuals. Immunity 52(6): 971-977.

20. Coperchini F, Chiovato L, Croce L, Magri F, Rotondi M (2020) The cytokine storm in COVID-19: An overview of the involvement of the chemokine/chemokine-receptor system. Cytokine Growth Factor Rev 53: 25-32.

21. Jose RJ, Manuel A (2020) COVID-19 cytokine storm: the interplay between inflammation and coagulation. Lancet Respir Med 8(6): 46-47.

22. Zhou Z, Ren L, Zhang L, Zhong J, Xiao Y, et al. (2020) Heightened Innate Immune Responses in the Respiratory Tract of COVID-19 Patients. Cell Host Microbe 27(6): 883-890.

23. Tay MZ, Poh CM, Rénia L, MacAry PA, Ng LFP (2020) The trinity of COVID-19: immunity, inflammation and intervention. Nat Rev Immunol 20(6): 363-374.

24. O'Neill LAJ, Netea MG (2020) BCG-induced trained immunity: can it offer protection against COVID-19? Nat
Rev Immunol 20(6): 335-337.

25. Brosch R, Gordon SV, Garnier T, Eiglmeier K, Frigui W, et al. (2007) Genome plasticity of BCG and impact on vaccine efficacy. Proc Natl Acad Sci 104(13): 5596-5601.

26. Covián C, Fernández-Fierro A, Retamal-Díaz A, Díaz FE, Vasquez AE, et al. (2019) BCG-Induced Cross-Protection and Development of Trained Immunity: Implication for Vaccine Design. Front Immunol 10: 2806.

27. Tanner R, Villarreal-Ramos B, Vordermeier HM, McShane $\mathrm{H}$ (2019) The Humoral Immune Response to BCG Vaccination. Front Immunol 10: 1317.

28. Moorlag SJCFM, Arts RJW, van Crevel R, Netea MG (2019) Non-specific effects of BCG vaccine on viral infections. Clin Microbiol Infect 25(12): 1473-1478.

29. Kleinnijenhuis J, van Crevel R, Netea MG (2015) Trained immunity: consequences for the heterologous effects of BCG vaccination. Trans R Soc Trop Med Hyg 109(1): 2935.

30. Covián C, Retamal-Díaz A, Bueno SM, Kalergis AM (2020) Could BCG Vaccination Induce Protective Trained Immunity for SARS-CoV-2?. Front Immunol.

31. Zhou T, Hu Z, Yang S, Sun L, Yu Z, et al. (2018) Role of Adaptive and Innate Immunity in Type 2 Diabetes Mellitus. J Diabetes Res 2018:7457269.

32. Curtis N, Sparrow A, Ghebreyesus TA, Netea MG (2020) Considering BCG vaccination to reduce the impact of COVID-19. Lancet 395(10236): 1545-1546. 